\title{
3.8-Gbit/s visible light communication (VLC) based on 443-nm superluminescent diode and bit- loading discrete-multiple-tone (DMT) modulation scheme
}

Hu, Fangchen, Holguin-Lerma, Jorge, Mao, Yuan, Shen, Chao, Sun, Xiaobin, et al.

Fangchen Hu, Jorge A. Holguin-Lerma, Yuan Mao, Chao Shen, Xiaobin Sun, Meiwei Kong, Tien Khee Ng, Boon S. Ooi, Nan Chi, "3.8-Gbit/s visible light communication (VLC) based on 443-nm superluminescent diode and bitloading discrete-multiple-tone (DMT) modulation scheme," Proc. SPIE 11307, Broadband Access Communication Technologies XIV, 113070H (31 January 2020); doi: $10.1117 / 12.2543983$

SPIE. Event: SPIE OPTO, 2020, San Francisco, California, United States 


\title{
3.8-Gbit/s visible light communication (VLC) based on 443-nm superluminescent diode and bit-loading discrete-multiple-tone (DMT) modulation scheme
}

\author{
Fangchen $\mathrm{Hu}^{1, \dagger}$, Jorge A. Holguin-Lerma ${ }^{2, \dagger}$, Yuan $\mathrm{Mao}^{2}$, Chao Shen ${ }^{2}$, Xiaobin $\mathrm{Sun}^{2}$, Meiwei Kong ${ }^{2}$, \\ Tien Khee $\mathrm{Ng}^{2}$, Boon S. Ooi ${ }^{2, \#}$, and Nan Chi ${ }^{1, *}$ \\ ${ }^{1}$ Key Laboratory for Information Science of Electromagnetic Waves (MoE), Department of \\ Communication Science and Engineering, Fudan University, Shanghai, China; \\ ${ }^{2}$ Photonics Laboratory, King Abdullah University of Science and Technology (KAUST), Thuwal \\ 23955-6900, Saudi Arabia; \\ †F. Hu and J. A. Holguin-Lerma contributed equally; \\ "boon.ooi@kaust.edu.sa, "nanchi@fudan.edu.cn
}

\begin{abstract}
There exists a demand for radiation-safe and high-speed communication systems available to public users in the fifthgeneration (5G) communication and beyond. In this regard, visible light communication (VLC) stands out offering multiGigabit-per-second (Gbit/s) data transmission, energy efficiency and illumination, while being free from electromagnetic interference. Here, we report a high-speed VLC link by using a 443-nm GaN-based superluminescent diode (SLD) and bit-loading discrete-multiple-tone (DMT) modulation. Analysis of the device characteristics and modulation parameters shows a feasible bit allocation of up to 256-QAM while obtaining up to $3.8 \mathrm{Gbit} / \mathrm{s}$ data rate. These results, together with the electro-optical properties of the SLD such as being droop-free, speckle-free and high-power, make it an attractive solution for the future of public communications and smart lighting, while complementing traditional fiber-based and millimeter-wave technology.
\end{abstract}

Keywords: Visible light communication (VLC), superluminescent diode (SLD), Gallium Nitride (GaN).

\section{INTRODUCTION}

The ultra-low-latency network beyond the fifth-generation $(5 \mathrm{G})$ will demand multi- Gigabit-per-second (Gbit/s) communication systems available to public users ${ }^{1}$ without the concerns of excessive radiation exposure. ${ }^{2,3}$ Under this context, visible light communication (VLC) have the potential to complement existing technologies by offering high-speed data transmission, energy efficiency, and integration to white lighting, while being free of electromagnetic interference. ${ }^{4}$ Up to date, a vast majority of the high-speed VLC systems have been demonstrated using light-emitting diode (LED) $)^{5-8}$ and laser diode (LD). ${ }^{9-11}$ A novel device combining advantages of both LD and LED, named superluminescent diode (SLD) has been explored towards VLC only on few occasions by our group ${ }^{12-15}$, however, additional investigation and development are needed to develop the full potential of SLDs towards high-speed VLC.

Visible light SLDs have shown attractive properties to a number of applications such as optical coherence tomography $(\mathrm{OCT})^{16}$ and augmented reality displays ${ }^{17}$. This is because of the SLD's unique combination of high-power, droop-free operation, low etendue, low temporal coherence, among others. One of the important applications of SLDs ${ }^{18}$, is their application to dual illumination and communication ${ }^{15}$, hence, the further improvement and research of SLDs towards VLC and lighting are important for technology and commercial endeavors.

The use of advanced modulation in VLC is set as the avenue for current development towards higher communication speed. Modulation schemes with high spectrum efficiency have been proposed for VLC applications such as discretemultiple-tone (DMT) modulation technique ${ }^{7,19}$. Such techniques maximize the data rate within a restricted power and bandwidth budget of the system. Additionally, equalization and bit-loading techniques enhance the optical system complementing the DMT scheme ${ }^{20}$.

Here, we report on the implementation of spectrally efficient DMT modulation applied to an SLD emitting at $443 \mathrm{~nm}$ with an optical bandwidth of $\sim 4.3 \mathrm{~nm}$ and an instantaneous peak optical power of $128 \mathrm{~mW}$. A data rate of $3.8 \mathrm{Gbit} / \mathrm{s}$ with a bit 
error ratio (BER) of 3.8 E-3 is demonstrated using bit-loading-DMT modulation scheme with post-equalization over a 30 $\mathrm{cm}$ free-space VLC channel for proof of concept. The achieved data rate relies on a device frequency bandwidth of up to $900 \mathrm{MHz}$, a system frequency bandwidth of $750 \mathrm{MHz}$, and a maximum quadrature amplitude modulation (QAM) order of 8 , leading to up to 256-QAM. By combining the optical characteristics of the SLD together with a spectrally efficient modulation scheme, we present a unique system that has the potential of be integrated into high-speed and user-oriented communication deployments in the future.

\section{PRINCIPLE}

\subsection{Superluminescent diode (SLD)}

A blue SLD was fabricated in a commercial c-plane group-III-Nitride-based laser diode structure by etching a $\sim 12^{\circ}$ tilted front facet (Fig. 1(a)). The device depicts a ridge waveguide with a length of $\sim 1 \mathrm{~mm}$ and a width of $15 \mu \mathrm{m}$. The $12^{\circ}$ tilted facet is chosen to reduce the reflectivity of the front facet and avoid lasing action, whereas the back facet is coated with a high reflective mirror following the design of a double-pass SLD reported by Alphonse ${ }^{21}$. Figure 1(b) shows the lightoutput-power - current - voltage (L-I-V) characteristics of the SLD as measured in continuous wave (CW) injection current with a laser diode test system (Keithley 2520) and a calibrated silicon photodetector in an integrating sphere (Labsphere). The device is mounted in a thermoelectric cooler (TEC, SaNoor-SN-LDM-T) to maintain a constant case temperature of $16^{\circ} \mathrm{C}$.

The I-V curve with a turn-on voltage of $\sim 3.5 \mathrm{~V}$ shows an almost linear I-V regime from $500 \mathrm{~mA}$ onwards (Fig. 1(b), black line), this linearity appeals potential for device modulation. The L-I curve (Fig. 1(b), blue line) increases exponentially due to the amplified spontaneous emission (ASE), leading to a droop-free performance as seen from the external quantum efficiency (EQE) plotted as a dashed line in Fig. 1(b). The instantaneous output power shows a maximum value of 128 $\mathrm{mW}$ as the current is increased to $1000 \mathrm{~mA}\left(\sim 6.7 \mathrm{kA} / \mathrm{cm}^{2}\right)$.
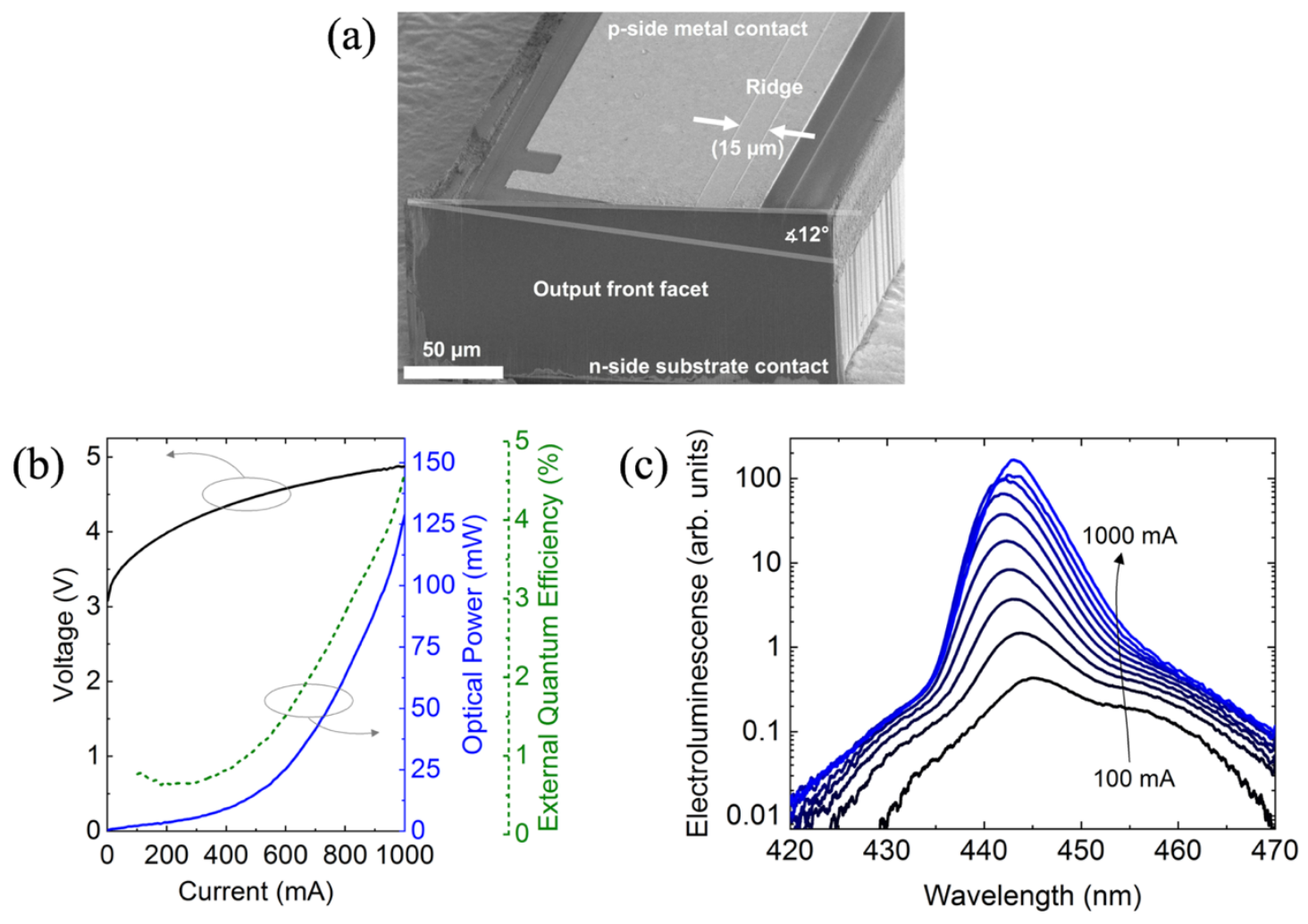

Figure 1. The SLD device and its electro-optical characteristics: (a) Scanning electron microscope image of the SLD showing the metal contact ( $\mathrm{p}$-side and n-side), the $15 \mu \mathrm{m}$ ridge-waveguide, and the $12^{\circ}$ tilted front facet. (b) Instantaneous light-outputpower - current - voltage (L-I-V) characteristics and calculated external quantum efficiency of the SLD (CW). (c) Electroluminescence (EL) spectra of the SLD under different CW injection current (100mA - 1000mA). 
The electroluminescence (EL) spectra characterized with an optical spectrum analyzer (Yokogawa AQ6373B) depicts characteristic SLD behavior as shown in Fig. 1(c). The optical bandwidth measured as the full width at half maximum (FWHM) decreases from $11 \mathrm{~nm}(100 \mathrm{~mA})$ to $7.8 \mathrm{~nm}(200 \mathrm{~mA})$, followed by an FWHM of $6.9 \mathrm{~nm}(300 \mathrm{~mA})$ down to 5 $\mathrm{nm}(900 \mathrm{~mA})$ and $4.3 \mathrm{~nm}(1000 \mathrm{~mA})$. Here, we can attribute the optical bandwidth narrowing to the ASE due to selective light amplification at the gain peak in the active region. Indeed, the ASE can be observed in Fig. 1(c) under injection currents as low as $100 \mathrm{~mA}$ (identified as the dominant peak at $445 \mathrm{~nm}$ ). The initial peak position located at $445 \mathrm{~nm}$ shows a relatively-small blue-shift to $442.3 \mathrm{~nm}(700 \mathrm{~mA})$ which is expected from the screening of the quantum-confined Stark effect at higher currents ${ }^{22}$ and the band-filling effect. The peak position increases again up to $443.3 \mathrm{~nm}(1000 \mathrm{~mA})$ due to self-heating in the device created by the higher CW injection current. Starting from $700 \mathrm{~mA}$, noticeable spectral distortions occur near the peak emission, these distortions are likely due to partial modal reflection at the front facet creating cavity modes $^{23}$. These are undesirable for certain applications such as the OCT, and are expected to be eliminated by using a different device design or an anti-reflection (AR) coating in the front facet, nonetheless, the AR coating could potentially lead to a lower output power ${ }^{18}$ which is important for lighting and displays applications.

\subsubsection{Frequency response}

The frequency response of the SLD in the range from $10 \mathrm{MHz}$ to $2 \mathrm{GHz}$ was measured using a network analyzer (NA, Agilent E8361C PNA) and the result is shown in Fig. 2, where a maximum frequency response of $\sim 600 \mathrm{MHz}$ at $-3 \mathrm{~dB}$, and $\sim 900 \mathrm{MHz}$ at $-10 \mathrm{~dB}$ are attained. The input signal of the SLD is generated from the NA and combined with the direct current (DC) bias from the power source (Keithley 2400) by using a bias-tee (Tektronix PSPL5580), while keeping the device in the TEC. The SLD's output light was collimated with a lens and projected over $30 \mathrm{~cm}$ to another lens focusing the light into an avalanche photodetector (APD, MenloSystem-APD210). The system is calibrated with an E-Cal module (Agilent 85093-60010).

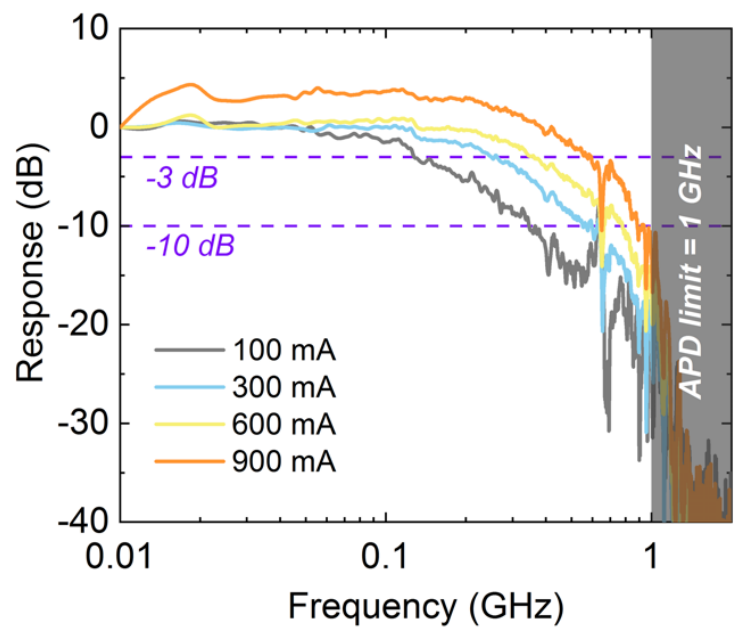

Figure 2. Frequency response of the SLD under various injection current showing a frequency bandwidth of up to $\sim 600 \mathrm{MHz}$ at $-3 \mathrm{~dB}$ and up to $\sim 900 \mathrm{MHz}$ at $-10 \mathrm{~dB}$.

\subsection{Bit-Loading Discrete-Multiple-Tone Modulation Scheme}

In order to take advantage of the full frequency resource of SLD, bit-loading-DMT modulation scheme is proposed to maximize the spectral efficiency. Bit-loading-DMT modulation scheme is a widely used and adaptive multi-carrier modulation technique which divides the frequency spectrum into multiple communication channels with a certain signalto-noise ratio (SNR). Considering the BER performance and the SNR, the suitable number of bits per symbol is assigned within each channel, which is also called bit-loading allocation. To modify the overall BER performance, we further shave some peak value of allocated QAM order, which builds bit-loading allocation with the shape of decreasing step and smooth transition. A typical bit-loading-DMT modulation schematic is shown in Fig. 3. Assuming that the length of the fast Fourier transform (FFT) is $N$, the generated bit-loading DMT signal $S_{b}$ of time $b$-th is defined as:

$$
S_{b}=\sum_{a=0}^{N-1} S_{a} e^{j 2 \pi \frac{b}{N} a}
$$


where $S_{a}$ is the $M$-QAM symbol on the $a$-th subcarrier. In detail, its distribution satisfies the Hermitian symmetry and its value depends on the SNR estimation of the certain channel.

\section{EXPERIMENTAL SETUP}

Figure 3 shows the experimental set up of the high-speed SLD-VLC system. The Pseudo-Random Binary Sequence (PRBS) of the signal was generated by MATLAB software and operated by the bit-loading allocation. The order of QAM mapping of each channel was decided by SNR estimation. Naturally, a higher SNR could accommodate a greater QAM order. Besides this, the signal needed zero-padding to avoid the negative effect of signal-signal beat interference (SSBI) and low-frequency noise after serial-parallel transformation. Then, the bit-loading-DMT signal was generated after Hermitian symmetry, up-sampling, inverse-FFT, adding the cyclic prefix $(\mathrm{CP})$ and the parallel-serial transformation. The generated signal was loaded into an arbitrary waveform generator (Tektronix-AWG70002A). Afterward, the electric signal was amplified by an electronic amplifier (MiniCircuits-ZHL-6A-S+) and coupled with DC bias by the bias-tee (TektronixPSPL5580) to drive the SLD. A TEC was used to avoid overheating of the SLD and to keep the output power constant. Collimated by two lenses and using a Neutral-Density (ND) filter, the light was received by the APD and resampled by an oscilloscope (Tektronix-DPO72004C) to do further offline signal processing.

At the receiver side, the resampled signal was transformed to the signal in the frequency domain by down-sampling and FFT. After that, Intra-symbol frequency-domain averaging (ISFA) was used to smoothing the channel and assist to do zero-forcing post-equalization to mitigate the linear noise. At last, we applied parallel-series transformation and QAM demodulation. It is important to mention that a binary phase-shift keying (BPSK)-DMT signal should be transmitted first to characterize the channel and get the SNR estimation.

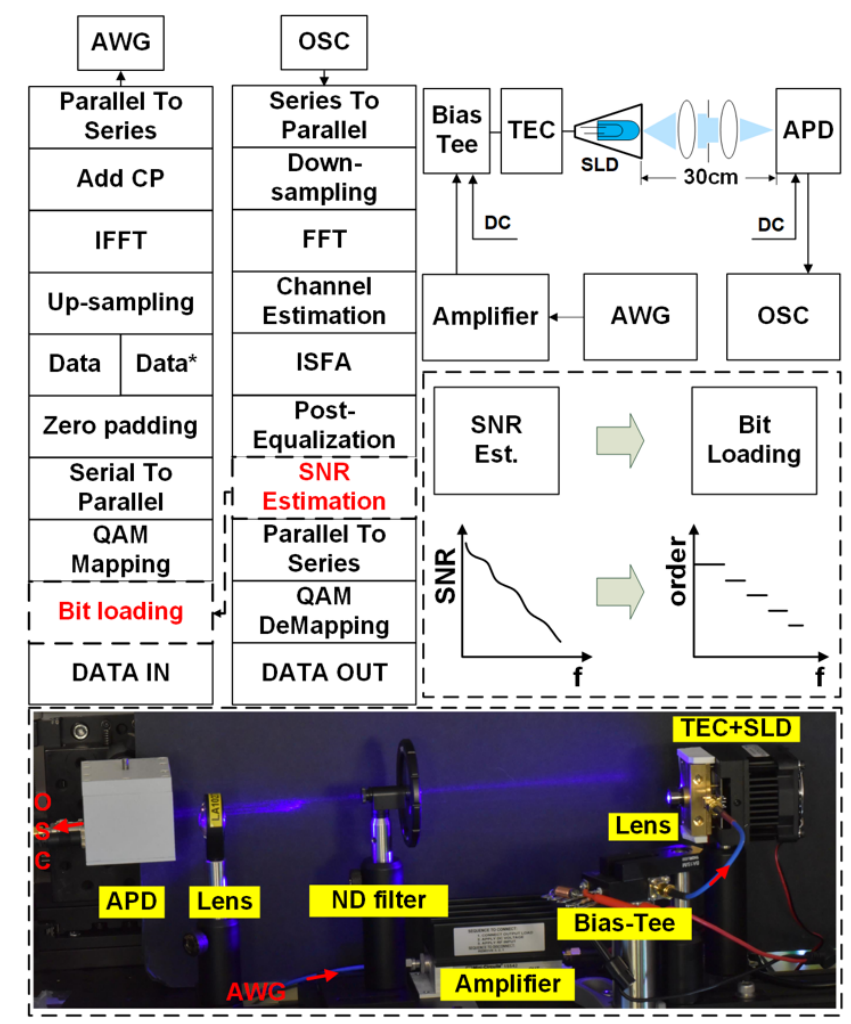

Figure 3. Upper: The schematic of bit-loading-DMT. Lower: Experimental set up of the VLC system based on SLD.

\section{RESULTS AND DISCUSSION}

For VLC, choosing a suitable value of injection current is a critical step to get the best electro-optical conversion and avoid the signal distortion. Thus, by using the bit-loading DMT scheme, we first measured the achievable data rate and BER performance under different injection current, with a reference fixed system channel bandwidth of $500 \mathrm{MHz}$ and a signal 
peak-to-peak voltage $\left(\mathrm{V}_{\mathrm{pp}}\right)$ of $0.5 \mathrm{~V}$. As shown in Fig. 4(a), the data rate increases from $1.53 \mathrm{Gbit} / \mathrm{s}$ to $2.67 \mathrm{Gbit} / \mathrm{s}$ when the current goes from $100 \mathrm{~mA}$ to $800 \mathrm{~mA}$. At $900 \mathrm{~mA}$, a lower data rate of $2.41 \mathrm{Gbit} / \mathrm{s}$ is at the same level as that of 700 $\mathrm{mA}(2.42 \mathrm{Gbit} / \mathrm{s})$, therefore, injection currents below $800 \mathrm{~mA}$ are preferred. It is important to highlight that having over $1.5 \mathrm{Gbit} / \mathrm{s}$ at such a low injection current of $100 \mathrm{~mA}$, indicates the feasibility of having Gbit/s-class communication at longer distances or in a scheme with reduced energy-budget.

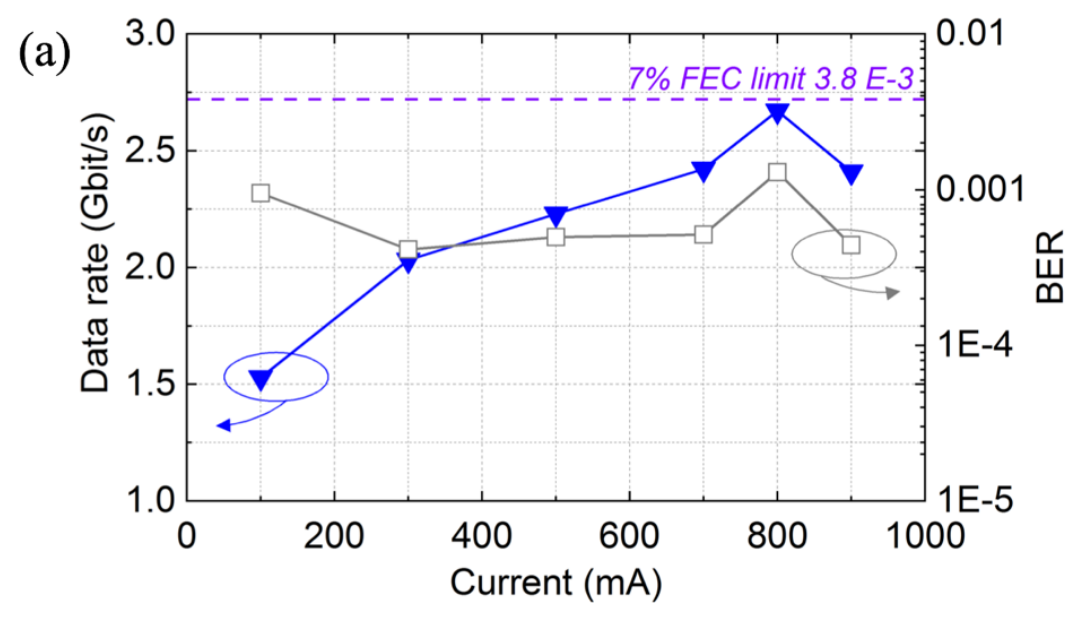

(b)

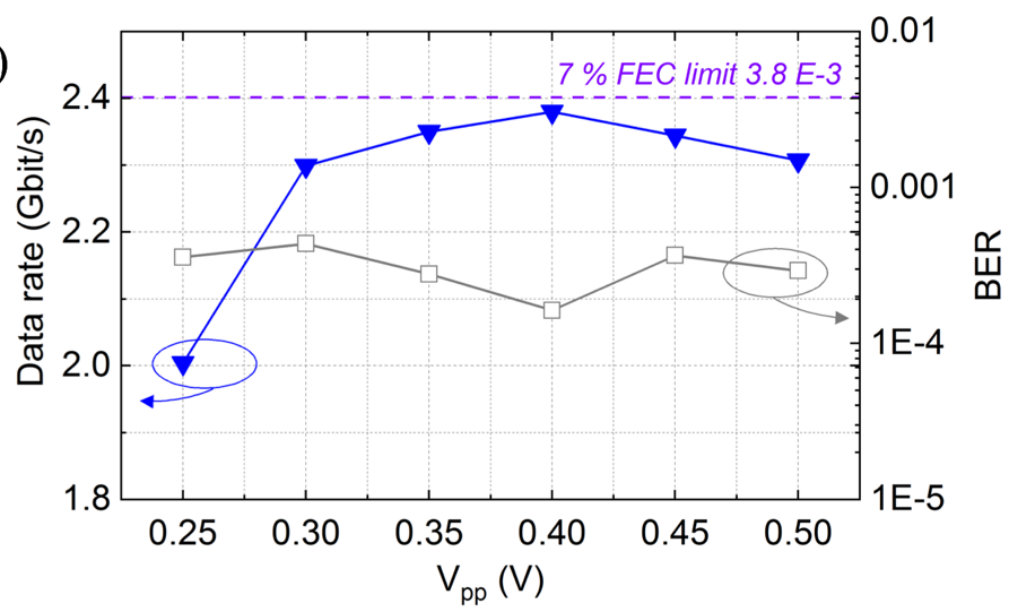

Figure 4. Achievable data rate and relative BER performance of the SLD versus (a) different injection current $(\mathrm{Vpp}=0.5 \mathrm{~V}$, freq. bandwidth $=500 \mathrm{MHz})$ and versus (b) different peak-to-peak voltage $\left(\mathrm{V}_{\mathrm{pp}}\right)(\mathrm{I}=700 \mathrm{~mA}$, freq. bandwidth $=500 \mathrm{MHz})$.

Next, we analyzed the system data rate and BER for a varying $\mathrm{V}_{\mathrm{pp}}$, a fixed injection current of $700 \mathrm{~mA}$, and bandwidth of $500 \mathrm{MHz}$ (Fig. 4(b)). When the $\mathrm{V}_{\mathrm{pp}}$ of the signal is growing from $0.25 \mathrm{~V}$ to $0.4 \mathrm{~V}$, the achievable data rate increases and the BER decreases. This is because the SNR of the system increases as $V_{p p}$ is increasing. After $0.4 \mathrm{~V}$, the data rate is decreased with a slightly higher BER. This is attributed to a bias point out of the optimal point, leading to non-linearities, distortion in the received signal, and decreased SNR. Thus, we select $0.4 \mathrm{~V}$ as the $\mathrm{V}_{\mathrm{pp}}$ value for further experimentation. In summary, the best values for biasing the SLD were determined to be $\sim 800 \mathrm{~mA}$ and $\sim 0.4 \mathrm{~V}$ ( $\mathrm{V}_{\mathrm{pp}}$ ).

We carried out experiments to explore the maximum achievable data rate under different frequency bandwidths with a fixed injection current of $800 \mathrm{~mA}$ and a $\mathrm{V}_{\mathrm{pp}}$ of $0.4 \mathrm{~V}$. As seen in Fig. 5, the highest data rate of $3.8 \mathrm{Gbit} / \mathrm{s}$ with a BER of $3.8 \mathrm{E}-3$ is obtained when the bandwidth is set to $750 \mathrm{MHz}$. 


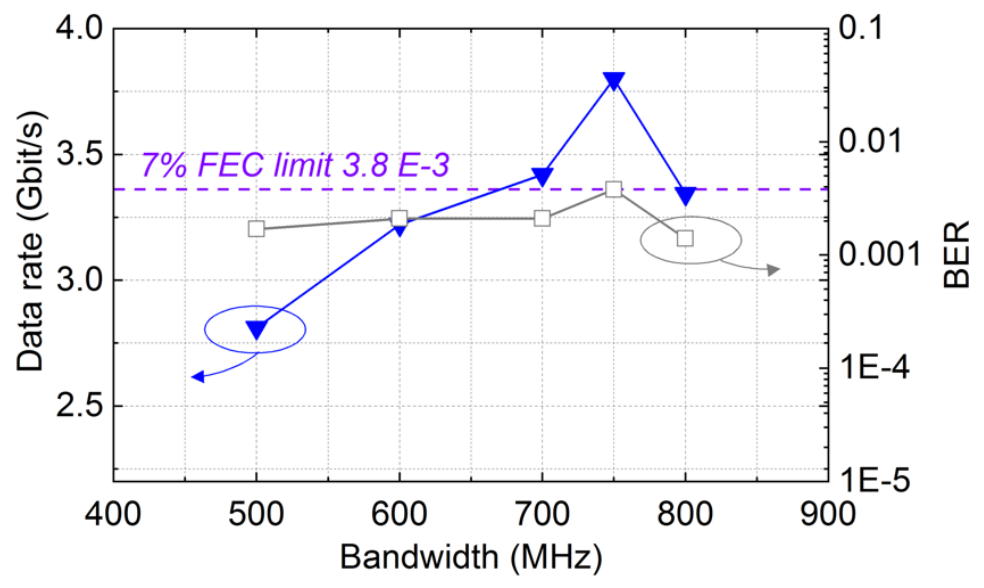

Figure 5. Achievable data rate and relative BER performance of the SLD versus different frequency bandwidth of bit loadingDMT signal.

(a)

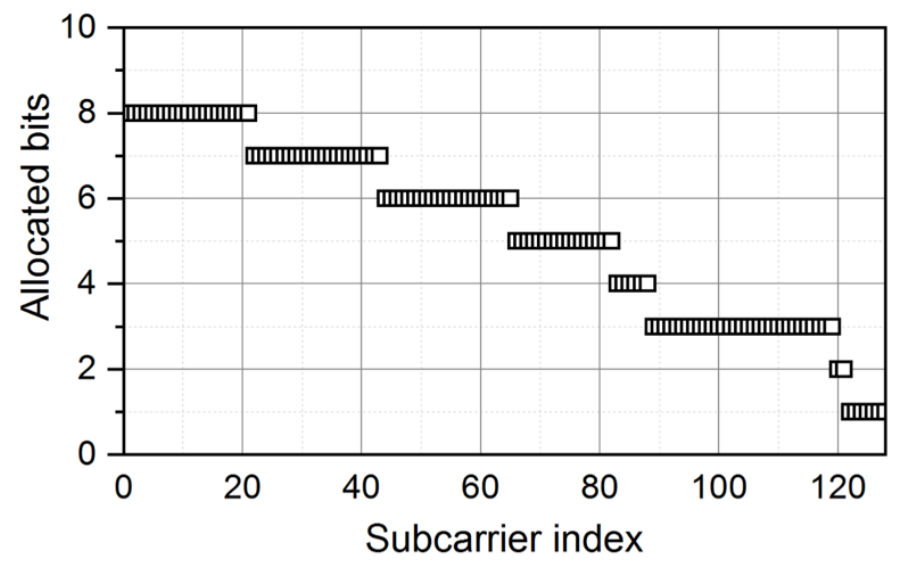

(b)

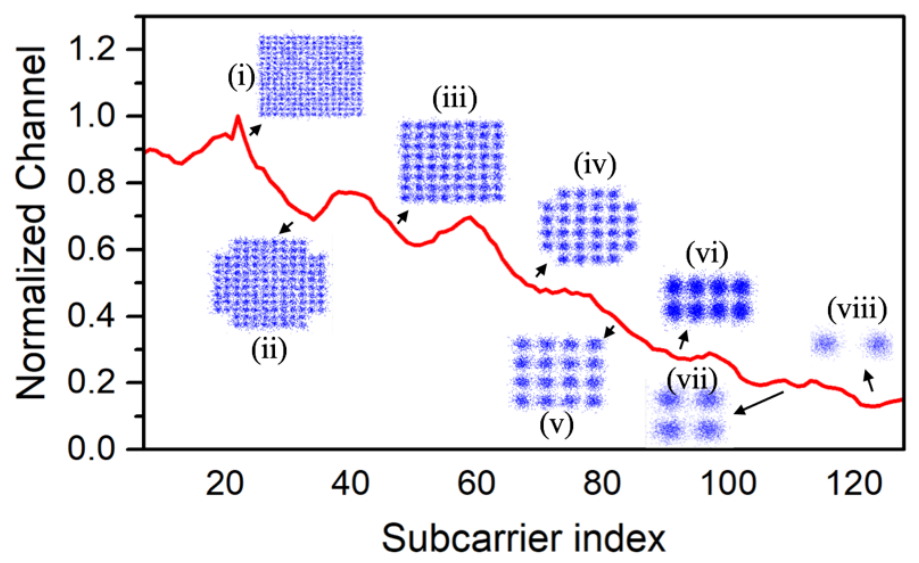

Figure 6. (a) Bit allocation and BER results with the corresponding performance of the adaptively QAM constellation. (b)(c) The frequency response and bit allocation at the highest data rate of $3.8 \mathrm{Gbit} / \mathrm{s}$. Constellation maps of (i) 256QAM, (ii) 128QAM, (iii) 64QAM. (iv) 32QAM (v) 16QAM (vi) 8QAM (vii) 4QAM (viii) BPSK at the highest data rate of $3.8 \mathrm{Gbit} / \mathrm{s}$

The description of the bit-loading allocation at the highest data rate of $3.8 \mathrm{Gbit} / \mathrm{s}$ is discussed in Fig.6. To adaptively deploy the suitable bit-loading allocation for the VLC channel, a series of DMT-4-QAM pilot signal is transmitted to measure the SNR of the channel. Through calculating the error vector magnitude (EVM) of the received signal in every subcarrier, the SNR response in the frequency domain can be estimated ${ }^{24}$ and depicted in Fig.6 (b). The theoretically maximum spectral 
capacity of every subcarrier can also be obtained from the estimated SNR in their own frequency range and the desired BER at 3.8 E-3 based on Equation 13 in Ref. 24. Thus, the value of the QAM order (allocated bits) for every subcarrier is the closest and smaller integer value to the theoretically maximum spectral capacity, shown in Fig.6 (a). To further prove the error-free transmission at the highest data rate, the constellation diagrams with different QAM orders are given in Fig. 6 (b), on which every constellation cluster can be clearly distinguished without severe overlapping problem. At the lowfrequency range, the high SNR can support up to 256-QAM signal transmission and the lowest QAM order is BSPK at the high-frequency range around $750 \mathrm{MHz}$, which means the channel has been fully exploited.

\section{CONCLUSIONS}

Gearing towards the potential implementation of a light-based multi-Gbit/s system available to public users in the future communication network, in this paper, we demonstrate a 3.8-Gbit/s VLC system based on a blue SLD with bit-loadingDMT modulation scheme. We presented the optical properties of the SLD, including high-power, broadband and droopfree emission, as well as the optimization of the bias point, $\mathrm{V}_{\mathrm{pp}}$, and frequency bandwidth for the communication system. The number of bits per symbol of QAM in the lower-order subcarriers is up to 8 (i.e. 256QAM) while fully utilizing the $750 \mathrm{MHz}$ bandwidth of the system. These results demonstrate the efficient application of bit-loading methodology to SLDbased VLC and set a further step into the future deployment of SLDs inside the illumination and communication technology environment.

\section{ACKNOWLEDGEMENT}

This work was partially supported by the National Key Research and Development Program of China (2017YFB0403603) and the NSFC project (No.61571133). This work was also supported by the King Abdullah University of Science and Technology (KAUST) funding BAS/1/1614-01-01, GEN/1/6607-01-01, REP/1/2878-01-01; KAUST equipment funding KCR/1/2081-01-01. This publication is also based upon work supported by the King Abdullah University of Science and Technology (KAUST) Office of Sponsored Research (OSR) under Award No. OSR-CRG2017-3417. TKN and BSO gratefully acknowledge funding from the King Abdulaziz City for Science and Technology (KACST) Grant no. R2-FP008.

\section{REFERENCES}

[1] Agiwal, M., Roy, A., and Saxena, N., "Next generation 5G wireless networks: a comprehensive survey," IEEE Communications Surveys \& Tutorials 18(3), 1617-1655 (2016).

[2] Russell, C.L., "5 G wireless telecommunications expansion: Public health and environmental implications," Environmental Research 165, 484-495 (2018).

[3] Di Ciaula, A., "Towards 5G communication systems: Are there health implications?," International Journal of Hygiene and Environmental Health 221(3), 367-375 (2018).

[4] Chi, N., Haas, H., Kavehrad, M., Little, T.D.C., and Huang, X.-L., "Visible light communications: demand factors, benefits and opportunities [Guest Editorial]," IEEE Wireless Communications 22(2), 5-7 (2015).

[5] Zhao, Y., Wang, F., Chi, N., Shi, J., Zhou, Y., Lu, X., Qiao, L., and Shi, M., "LED-based high-speed visible light communications," Proc. SPIE 10559, 105590I (2018).

[6] Bian, R., Tavakkolnia, I., and Haas, H., "15.73 Gb/s Visible Light Communication with Off-the-Shelf LEDs," Journal of Lightwave Technology 37(10), 2418-2424 (2019).

[7] Zhou, Y., Zhu, X., Hu, F., Shi, J., Wang, F., Zou, P., Liu, J., Jiang, F., and Chi, N., "Common-anode LED on a Si substrate for beyond $15 \mathrm{Gbit} / \mathrm{s}$ underwater visible light communication," Photonics Research 7(9), 1019-1029 (2019).

[8] Rajbhandari, S., McKendry, J.J.D., Herrnsdorf, J., Chun, H., Faulkner, G., Haas, H., Watson, I.M., O’Brien, D., and Dawson, M.D., "A review of gallium nitride LEDs for multi-gigabit-per-second visible light data communications," Semiconductor Science and Technology 32(2), 023001 (2017).

[9] Guo, Y., Alkhazragi, O., Kang, C.H., Shen, C., Mao, Y., Sun, X., Ng, T.K., and Ooi, B.S., "A tutorial on laser-based lighting and visible light communications: device and technology [Invited]," Chinese Optics Letters 17(4), 040601 (2019).

[10] Tsai, C.-T., Cheng, C.-H., Kuo, H.-C., and Lin, G.-R., "Toward high-speed visible laser lighting based optical wireless communications," Progress in Quantum Electronics 67(1), 100225 (2019).

[11] Lu, H.-H., Li, C.-Y., Chen, H.-W., Ho, C.-M., Cheng, M.-T., Yang, Z.-Y., and Lu, C.-K., “A 56 Gb/s PAM4 VCSEL- 
Based LiFi Transmission With Two-Stage Injection-Locked Technique,” IEEE Photonics Journal 9(1), 1-8 (2017).

[12] Shen, C., Lee, C., Ng, T.K., Nakamura, S., Speck, J.S., DenBaars, S.P., Alyamani, A.Y., El-Desouki, M.M., and Ooi, B.S., "High-speed 405-nm superluminescent diode (SLD) with 807-MHz modulation bandwidth," Optics Express 24(18), 20281-20286 (2016).

[13] Shen, C., Ng, T.K., Lee, C., Leonard, J.T., Nakamura, S., Speck, J.S., Denbaars, S.P., Alyamani, A.Y., El-Desouki, M.M., et al., "Semipolar InGaN-based superluminescent diodes for solid-state lighting and visible light communications," Proc. SPIE 10104, 101041U (2017).

[14] Alatawi, A.A., Holguin-Lerma, J.A., Kang, C.H., Shen, C., Subedi, R.C., Albadri, A.M., Alyamani, A.Y., Ng, T.K., and Ooi, B.S., "High-power blue superluminescent diode for high CRI lighting and high-speed visible light communication," Optics Express 26(20), 26355 (2018).

[15] Shen, C., Holguin-Lerma, J.A., Alatawi, A.A., Zou, P., Chi, N., Ng, T.K., and Ooi, B.S., "Group-III-Nitride Superluminescent Diodes for Solid-State Lighting and High-Speed Visible Light Communications," IEEE Journal of Selected Topics in Quantum Electronics 25(6), 2000110 (2019).

[16] Goldberg, G.R., Ivanov, P., Ozaki, N., Childs, D.T.D., Groom, K.M., Kennedy, K.L., and Hogg, R.A., "Gallium nitride light sources for optical coherence tomography," Proc. SPIE 10104, 101041X (2017).

[17] Primerov, N., Dahdah, J., Gloor, S., von Niederhäusern, T., Matuschek, N., Castiglia, A., Malinverni, M., Mounir, C., Rossetti, M., et al., "A compact red-green-blue superluminescent diode module: A novel light source for AR microdisplays," Proc. SPIE 11062, 110620F (2019).

[18] Kafar, A., Stanczyk, S., Schiavon, D., Suski, T., and Perlin, P., "Review-Review on Optimization and Current Status of (Al,In)GaN Superluminescent Diodes," ECS Journal of Solid State Science and Technology 9(1), 015010 (2020).

[19] Stepniak, G., Schuppert, M., and Bunge, C.-A., "Advanced Modulation Formats in Phosphorous LED VLC Links and the Impact of Blue Filtering," Journal of Lightwave Technology 33(21), 4413-4423 (2015).

[20] Chow, P.S., Cioffi, J.M., and Bingham, J.A.C., "A practical discrete multitone transceiver loading algorithm for data transmission over spectrally shaped channels," IEEE Transactions on Communications 43(2/3/4), 773-775 (1995).

[21] Alphonse, G.A., "Design of high-power superluminescent diodes with low spectral modulation," Proc. SPIE 4648, $125-138$ (2002).

[22] Kafar, A., Stanczyk, S., Grzanka, S., Pieniak, K., Suski, T., and Perlin, P., "Screening of quantum-confined Stark effect in nitride laser diodes and superluminescent diodes," Applied Physics Express 12(4), 044001 (2019).

[23] Kopp, F., Lermer, T., Eichler, C., and Strauss, U., "Cyan superluminescent light-emitting diode based on InGaN quantum wells," Applied Physics Express 5, 082105 (2012).

[24] Shafik, R.A., Rahman, M.S., and Islam, A.R., "On the Extended Relationships Among EVM, BER and SNR as Performance Metrics,” 4th Int. Conf. Electr. Comput. Eng., ICECE 2006, 408-411 (2006). 